\title{
Connection Parameters of Heavy-tailed Operational Risk Measurement Model and Management Model
}

\author{
Meiling He ${ }^{1}$, Shutao Qing ${ }^{2}$, Jianming Mo ${ }^{1}, \quad$ Xiang $\mathbf{G a o}^{3}$ \\ ${ }^{I}$ Chinese Finance Research Institute, Southwestern University of Finance and Economics, Chengdu \\ 610074, China \\ ${ }^{2}$ Hunan provincial Party School Department of Economics, Changsha 410006, China \\ ${ }^{3}$ School of International Business Administration, Shanghai University of Finance and Economics, \\ Shanghai 200433, China
}

Received 26 April 2016

Accepted 2 July 2016

\begin{abstract}
In order to connect the heavy-tailed operational risk measurement model with management model, a model identifying the crucial supervising parameters of operational risk is built after the heavy-tailed operational VaR's sensitivity is theoretically researched by the elasticity analysis method. Further, the analysis of model application is illustrated with a numerical example. The crucial supervising parameters connect the operational risk measurement model and management model, which make the operational risk management frameworks to be a complete system. And a dynamical supervising system of operational risk is established. This research in theory improves the application of loss distribution approach to the operational risk measurement and management.
\end{abstract}

Keywords: operational risk; supervising parameters; elasticity theory; operational VaR

\section{Introduction}

Operational risk management is an integrated frame work which includes risk identification, measurement, analysis, monitoring, mitigation and economic capital allocation. In such management framework, how to combine risk management model with measurement model is a major issue. However, current operational risk mainly aims to calculate capital reserve precisely and monitor management effect by directly observing the variation of the capital reserve. Thus, operational risk measurement and management are practically independent system, which leads to lack efficiency and target in proposing measures for risk management.

Generally speaking, the aim of risk measurement is not restricted to calculate regulatory capital, more importantly, to provide valid foundation for making management regulations, thereby reducing the whole risks.

*National Natural Science Foundation of China(71171167, 71671144, 71373213, 71301130, 71501117, 13YJC790024)
However, it is relatively late for us to attach importance to operational risk in theory and practice field. It wasn't until June 2004 that new Basel Accord was issued and since then operational risk has been included in the regulatory system. Therefore, the framework of operational risk management is still in the process of improving and burgeoning. And how to find connection parameters to combine measurement model with management model is still a problem which has not been solved.

Existing literatures regard Bayesian network as the best model to manage operational risk. The parameters of the nodes in Bayesian net work represent the characteristic parameters of the Loss Distribution Approach. The methodology of choosing network nodes is divided into three categories: (1) regard key risk indicators (e.g., employee turnover rates) as nodes; (2) consider loss frequency and loss severity caused by loss events as nodes; (3) define characteristic parameters of the loss distribution as nodes. Actually, three types of 
nodes reflect operational risk from diverse perspectives. While key risk indicators are able to reflect the change of the operational risk, they still cannot reflect the measurement changes in results. Although the mathematical expectation of loss frequency and severity distribution can reflect measure changes directly, under the condition that loss severity distribution is heavy-tailed, loss severity distribution may not have mathematical expectation. Moreover, some empirical researches have proved that loss severity distribution has significantly heavy-tail characteristic, meaning that under some loss severity distribution, internet nodes are unavailable. Fortunately, no matter how loss distribution changes, characteristic parameters still exist and directly reflect the change result of measurement. Consequently, in Bayesian model, using key risk indicator or expectation of loss frequency and severity as nodes do have some drawbacks, therefore it is appropriate to take characteristic parameter as internet nodes.

Previous literatures have found that the object that the operational risk management target at is exactly the characteristic parameter of loss distribution. Chapelle and Grama et al (2008) found that varied operational risk management measures affect different loss distribution: some management measures(e.g., "Dashboard") solely influence loss frequency distribution, some management measures (e.g., "Rapid reaction") solely influence loss severity distribution, while some other management measures (e.g., "Audit tracking", "Business line") have both influence on loss frequency distribution and loss severity distribution and thereby achieving regulation deviation. Further more, that means, disparate management measures have different impact on different characteristic parameters and operational risk management is essentially to govern characteristic parameter. In order to maximize management efficiency, the chief management objects should be the characteristic parameter which exerts enormous effect on operational VaR. These parameters can be identified only by measurement model.

Can we identify the key management parameters by measurement model? At present, financial institutes mainly use Loss Distribution Approach to measure operational risk. Under that method, operational $\mathrm{VaR}$ is formulated by loss severity distribution and loss frequency distribution. However, with LDA, we can only calculate operational $\mathrm{VaR}$ by convolution calculation formula, sparse vector method and Panjer recursion etc. Therefore, analytical solution of the operational value at Risk is non-existent, which results in two problems. For one thing, we do not know which parameters exert the biggest impact on operational $\mathrm{VaR}$, so management target is uncertain. For another thing, when one parameter changes, we are unable to tell whether capital reserve estimate changes and the direction it changes to. Consequently, we fail to evaluate our risk management measures that mean operational risk measurement cannot afford useful information for risk management so that blindness exists in management.

The existing of analytical solution of operational $\mathrm{VaR}$ is a prerequisite for addressing the above problems. Bocker and KlÄuppelberg (2005), Bocker and Sprittulla (2006) and Bocker (2006) have found that, with LDA, when it comes to the general distribution, the analytical solution to operational VaR is not existing. But as for the heavy-tailed risk measurement, the value of the tail does have analytical solution. Operational VaR exactly satisfies the condition: New Basel Accord stipulated that, the regulatory capital for operational risk is calculated by VaR at a confidence level $99.9 \%$. It is actually tail value under high confidence level as well as heavy-tailed distribution. Therefore, there is an analytical solution to heavy-tailed operational VaR.

The analytical solution of operational VaR combines characteristic parameters with operational VaR directly. And thereby it is likely to discuss the sensitivity of capital reserve estimate to changes in characteristic parameter and its change trend. By doing so, we can identify the parameter, which influences capital reserve most as the key management parameter of the elasticity, and furnish references for management measures. That is, the key management parameters are used to synthesis measurement model with management model. Measurement model not only affords management object to management model and take the function of effect surveillance of management model, but also provides valid ground for management measures revision. Hence, the key management parameters technically become connection parameters, 
turning the framework of operational risk management into a cycling system.

Literature 10 manifests that, when we use Generalized Extreme Value Distributions to model severity distribution, some operational loss events compiles with Weibull distribution. So our article discusses the combination parameter for measurement model and management model under that distribution. Literature 16 finds that, if loss severity distribution follows Weibull distribution, we can get the analytical solution of the operational VaR. Moreover, this article investigates the confidence level and sensitivity of operational VaR using computer simulation. Based on this, our paper assumes the loss severity distribution follows Weibull distribution, and explores the key management parameters by analyzing the sensitivity of operational $\mathrm{VaR}$ to characteristic parameter. First, our paper derives the analytical solution of the operational VaR under high confidence level. We probe into the parameter elasticity of operational $\mathrm{VaR}$ and discrimination model is established by operational risk measurement model and management model. Finally, we prove its validity by numerical analysis.

Operational risk is embedded in all kinds of financial institutions and economic activities. Compared with conventional market risks and credit risks, the management of operational risk is harder. Partially, it cannot be eliminated by selling positions. Our article creatively comes up with a valuable model to manage operational risk. And by identifying and researching the key parameters of operational risk, financial institutions are able to curb operational risk effectively and manage operational risk in some sense.

\section{Measurement of operational VaR}

Using LDA to measure heavy-tailed operational risk, one can derive the analytical solution of the Operational $\operatorname{VaR}(O p \operatorname{VaR}(\alpha))$ as follows at high confidence level:

$$
O p V_{\Delta a}(\alpha) \cong F^{-1}\left(1-\frac{1-\alpha}{E N(\Delta t)}\right)
$$

where $\Delta t$ is the target horizon, $\alpha$ is a confidence level close to $1, \mathrm{~F}(\cdot)$ is cumulative function, $E N(\Delta t)$ is the expected loss frequency during $\Delta t$. For convenience, in the following, we use $\mu$ to replace $E N(\Delta t)$ for simplification.

Based on the nature of cumulative distribution functions, equation (1) implies $0 \leq 1-(1-\alpha) / \mu \leq 1 \quad$ and consequently $\mu /(1-\alpha) \geq 1$.

According to the numerical results for operational risk loss severity distribution in literature 10, we assume the loss severity follows a Weibull distribution:

$$
\begin{aligned}
& F_{w}(x)=1-\exp \left[-\left(\frac{x}{\theta_{w}}\right)^{\xi_{w}}\right] \\
& x>0, \xi_{w}>0, \theta_{w}>0
\end{aligned}
$$

where $\mathrm{x}$ denotes loss severity, $\theta_{w}$ and $\xi_{w}$ are the shape and scale parameters of the Weibull distribution respectively. Plug equation (4) into equation (2) to obtain

$$
\begin{gathered}
O p \operatorname{VaR}_{\Delta t}(\alpha)_{w} \cong \theta_{w}\left(\ln \frac{\mu}{1-\alpha}\right)^{\frac{1}{\xi_{w}}} \\
\xi_{w}>0, \theta_{w}>0, \mu \geq 0
\end{gathered}
$$

Since $\frac{\mu}{1-\alpha} \geq 1$, then $\ln \frac{\mu}{1-\alpha} \geq 0$, operational risk restores normalcy, $\operatorname{OpVaR}_{\Delta t}(\alpha)_{w} \geq 0$, equation(3) makes sense. When $\mu /(1-\alpha)=1$, operational risk is non-existent, $O p V a R_{\Delta t}(\alpha)_{w}=0$, which indicates that the financial institution in question has ceased business operations. Only on the condition that $\mu /(1-\alpha) \geq 1$, we can discuss the key management indicators for operational risk. From equation (3) we know that at some certain confidence level $\alpha$, the estimated $\operatorname{Op} \operatorname{VaR}(\alpha)$ only depends on characteristic parameters $\left(\theta_{w}, \xi_{w}\right.$ and $\left.\mu\right) . \theta_{w}$ and $\xi_{w}$ are the shape and scale parameters of the Weibull distribution respectively, while $\mu$ is the characteristic parameter of loss frequency distribution. Different management measures have different impact on loss frequency distribution as well as severity distribution. To be specific, policy effect on characteristic parameters differs, so the influence on $\operatorname{Op} \operatorname{VaR}(\alpha)$ differs. Consequently, the parameter which has greatest effect on $\operatorname{Op} \operatorname{VaR}(\alpha)$ is the key management indicator for operational risk management. Naturally, it is the connection parameter of measurement model and management model as well. In the following, we will discuss how to identify these parameters.

\section{Connection parameters of measurement model and management model}

\subsection{Theoretical model}

Connection parameters of measurement model and management model are the key management parameters for operational risk. In order to manage risk comprehensively, we need to identify two kinds of key parameters: First, distinguish main influential factors of 
operational risk. There is no doubt that these influential factors can provide references for authorities to draw on when setting rules. Second, we need to distinguish the key parameters which affect operational VaR's sensitivity enormously. By monitoring and predicting the possible change of these key parameters, we can come up with possible direction for regulators to revise their measurements. In the following, let us investigate it through the sensitivity analysis $O p \operatorname{VaR}(\alpha)$.

For different characteristic parameters $\left(\theta_{w}, \xi_{w}\right.$ and $\left.\mu\right)$, the numerical size and variation range differs greatly. The variation of $O p \operatorname{VaR}(\alpha)$ (i.e., $\Delta O p \operatorname{VaR}(\alpha)$ ) caused by absolute variation of $\theta_{w}, \xi_{w}$ and $\mu\left(\Delta \theta_{w} 、 \Delta \xi_{w}\right.$ 、 $\Delta \mu$ ), cannot fully reflect the influence of characteristic parameters to the sensitivity of $O p \operatorname{VaR}(\alpha)$ s. Only the percentage change in the $(\triangle O p \operatorname{VaR}(\alpha) / O p \operatorname{VaR}(\alpha) \quad) \operatorname{Op} \operatorname{VaR}(\alpha)$ in response to one percent change in the characteristic parameter $\left(\Delta \xi_{w} / \xi_{w} 、 \Delta \theta_{w} / \theta_{w}\right.$ 、

$\Delta \mu / \mu)$ does accurately denotes the sensitivity of $O p \operatorname{VaR}(\alpha)$ relating to $\theta_{w} \xi_{w}$ and $\mu$.According to elastic theory, elasticity can be quantified as the ratio of the percentage change in one variable to the percentage change in another variable, while the latter variable has influence on the former. Therefore, we can use parameter elasticity of $O p \operatorname{VaR}(\alpha)$ as a proxy of the $O p \operatorname{VaR}(\alpha)$ sensitivity with respect to characteristic parameters.

Definition the elasticity of $\operatorname{Op} \operatorname{VaR}(\alpha)$ in response to $\theta_{w}, \xi_{w}, \mu$

$$
\begin{aligned}
E_{\xi_{w}} & =\lim _{\Delta \xi_{w} \rightarrow 0} \frac{\Delta O p \operatorname{VaR}(\alpha) / O p \operatorname{VaR}(\alpha)}{\Delta \xi_{w} / \xi_{w}} \\
E_{\theta_{w}} & =\lim _{\Delta \theta_{w} \rightarrow 0} \frac{\Delta O p \operatorname{VaR}(\alpha) / O p \operatorname{VaR}(\alpha)}{\Delta \theta_{w} / \theta_{w}} \\
E_{\mu} & =\lim _{\Delta \mu \rightarrow 0} \frac{\Delta O p \operatorname{VaR}(\alpha) / O p \operatorname{VaR}(\alpha)}{\Delta \mu / \mu}
\end{aligned}
$$

Plug equation (3) into the definition we obtain:

$$
\begin{gathered}
E_{\theta_{w}}=1 \\
E_{\xi_{w}}=-\ln \left(\ln \frac{\mu}{1-\alpha}\right)^{\xi_{w}^{-1}} \\
E_{\mu}=\left(\xi_{w} \ln \frac{\mu}{1-\alpha}\right)^{-1}
\end{gathered}
$$

Equation (4) denotes that, scale parameter elasticity of $O p \operatorname{VaR}(\alpha)$ is unit elasticity. i.e., $\operatorname{Op} \operatorname{VaR}(\alpha)$ always changes $1 \%$ while $\theta$ changes $1 \%$.Equation(5)and(6) imply that shape parameter elasticity of $\operatorname{Op} \operatorname{VaR}(\alpha)$ and frequency parameter elasticity of $O p \operatorname{VaR}(\alpha)$ have no relationship with scale parameter, which means scale parameter has no impact on the $O p \operatorname{VaR}(\alpha)$ sensitivity of characteristic parameters of $\xi$ and $\mu$. But shape parameter and frequency parameter do have some influence. The reason for this is that the operational risk is influenced by both loss severity distribution and loss frequency distribution. In the loss severity distribution, shape parameter denotes the thickness and length of the tail, (i.e., it decides the tail risk). In the loss frequency distribution, operational risk is decided by loss frequency. Scale parameters and frequency parameters respectively represent the influence of severity distribution and frequency distribution. If the frequency parameter is the key parameter, then $\operatorname{Op} \operatorname{VaR}(\alpha)$ is mostly influenced by loss frequency distribution. Thus, identifying the key parameter for operational risk actually equals to selecting parameter from shape parameter and frequency parameter. Furthermore, we can distinguish the key influential indicator. In the following, we list the general methodology to judge the key management parameters.

Before we come up with our propositions, we would better reclaim our assumptions: The financial institutions we concerned about use Loss Distribution Approach to measure operational risk and do not cease business operations. Loss severity distribution of operational risk complies with Weibull distribution.

Proposition 1 Under the previous assumption, we find following rules

$$
E_{\mu}>0 \quad ; \quad \text { as } \quad 0<\ln \frac{\mu}{1-\alpha} \leq 1 \quad \text {, }
$$$$
E_{\xi_{w}} \geq 0, \text { as } \ln \frac{\mu}{1-\alpha}>1, E_{\xi_{w}}<0
$$

(ii) as $\alpha \rightarrow 1, E_{\xi_{w}} \rightarrow-\infty, E_{\mu} \rightarrow 0$

(iii)as $\left|-\ln \frac{\mu}{1-\alpha} \ln \left(\ln \frac{\mu}{1-\alpha}\right)\right| \geq 1 \quad\left|E_{\xi_{w}}\right| \geq E_{\mu}$, otherwise, $\left|E_{\xi_{w}}\right|<E_{\mu}$.(see proof in Appendix A)

From proposition 1(i), we know that two possible factors contribute to the risk magnifying one is the increasing of frequency parameters, 
that mean though loss severity is not huge, increasing loss frequency would lead to overall loss increase. The other is the variation of the shape parameter: when $\ln \frac{\mu}{1-\alpha}>1$, as shape parameter increases, the tail of the operational risk is thinner, and operational risk increases. Conversely, if shape parameter decreases, operational risk decreases. Specifically, in order to reduce operational risk, the change direction of shape parameter hinges on different operational condition. Hence, in the reality to manage operational risk, if shape parameter is the key management parameter, we should judge the case first. Then we can fix the change direction of the parameter and make management measures to achieve it.

Further, according to proposition 1(ii), theoretically, when confidence level $\alpha$ approximates to 1 (confidence level is very high), shape parameter would exert enormous influence on operational risk (tend to infinity), while frequency parameter has little influence on it (tend to zero)

According to proposition 1(iii), the different influence two parameters exert on operational risk can be represented by the criterion $|\ln [\mu /(1-\alpha)] \ln \ln [\mu /(1-\alpha)]|$. Set $\Delta E=-\ln [\mu /(1-\alpha)] \ln \ln [\mu /(1-\alpha)] \quad$. Given $|\Delta E|>1$, shape parameter's influence is greater than frequency parameter. As $\alpha$ increases, $|\Delta E|$ increases and the diversity becomes larger. Given $|\Delta E|<1$, shape parameter's influence is less than frequency parameter. As $\alpha$ increases, $|\Delta E|$ increases and the diversity becomes smaller. When $\alpha$ is fixed, discriminant is only decided by $\mu$,i.e., the diversity between $\left|E_{\xi_{w}}\right|$ and $E_{\mu}$ is decided by $\mu$. Set $\alpha=99.9 \%$, Figure 1 draws how discriminant changes against the frequency parameter $\mu$.

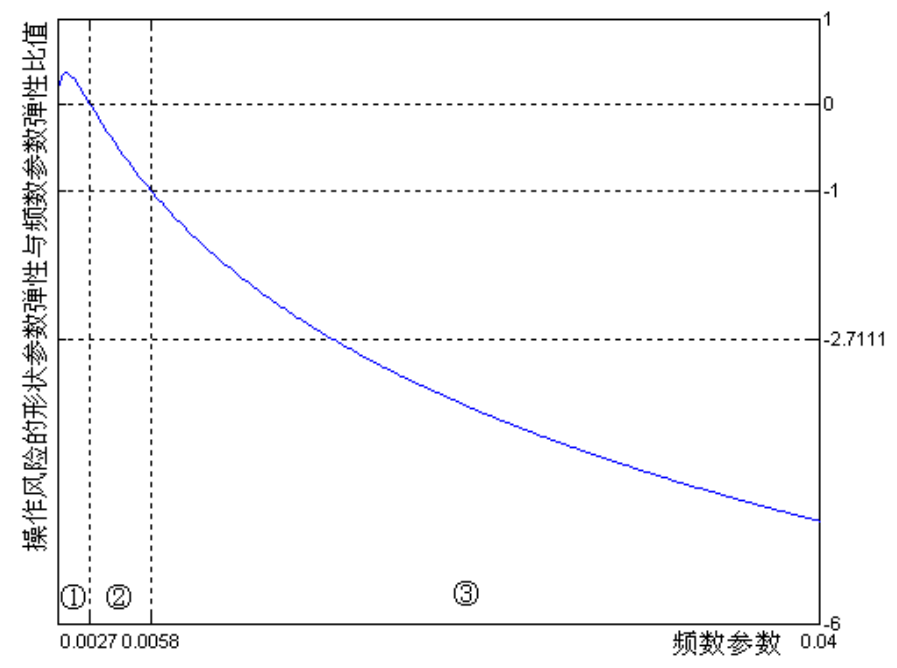

Fig.1. the ratio of $E_{\xi_{w}}$ to $E_{\mu}$

As $\frac{\mu}{1-\alpha}>1$, then $\mu>0.001$. Therefore figure 1 regards $\mu=0.001$ as the starting point of abscissa.

Given $\Delta E=0$, we can get $\mu=0.0027, E_{\xi_{w}}=0$, i.e., shape parameters has no influence on operational risk. Given $\Delta E=-1$, we can get $\mu=0.0058$, i.e., as shape parameter increases, operational risk decreases and shape parameter and frequency parameter have the same influence on it. Therefore, there exist three regimes for the relationship between $\mu$ and $|\Delta E|$.

According to figure1:

In section (1), given $0.001<\mu<0.0027$, then $\Delta E>0$ and $|\Delta E|<1 \quad$ We know $E_{\xi_{w}}>0$ and $E_{\xi_{w}}<E_{\mu}$. As shape parameter (or frequency parameter) increases, operational risk increases, but frequency parameter exerts greater influence than shape parameter. Under this condition, frequency parameter is the main 
factors to affect operational risk. i.e., loss frequency distribution is the main management object

In section (2), given $0.0027<\mu<0.0058$, then $\Delta E<0,|\Delta E|<1$. We know $E_{\xi_{w}}<0\left|E_{\xi_{w}}\right|<E_{\mu}$. As shape parameter decreases (or frequency parameter increases), operational risk increases, but frequency parameter exerts greater influence than shape parameter. Under this condition, frequency parameter is the main factors to affect operational risk. i.e., loss frequency distribution is the main management object.

In section (3), given $\mu>0.0058$ then $\Delta E<0$ and $|\Delta E|>1$ We know $E_{\xi_{w}}<0$ and $\left|E_{\xi_{w}}\right|>E_{\mu}$. As shape parameter decreases (or frequency parameter increases), operational risk increases, but shape parameter exerts greater influence than frequency parameter. Under this condition, shape parameter is the main factors to affect operational risk. i.e., loss severity distribution is the main management object. As we can see in the figure1, with $\mu$ increasing, the curve shifts to the right bottom of the picture and discriminant becomes larger, i.e., the difference between $\left|E_{\xi_{w}}\right|$ and $E_{\mu}$ becomes larger. Therefore, shape parameter plays a significantly pivotal role in risk management.

Consequently, according to different operational risk condition, financial institutes are supposed to firstly judge different section of loss distribution and then find out the key management indicator by proposition 1.This proposition not only provides foundation for setting and revising management measures, but also can be used to check the effect of the measures.

From equation(5) (6), under the certain confidence level, the elasticity of $\operatorname{Op} \operatorname{VaR}(\alpha)$ changes as shape parameter and frequency parameter change, and therefore relative size of $E_{\xi_{w}}$ and $E_{\mu}$ varies. Owing to this, the key management parameter of the operational risk can change from shape parameter to frequency parameter or change from frequency parameter to shape parameter. Further on, we will go ahead to introduce proposition 2 to discuss the changing rule between $E_{\xi_{w}}$ and $E_{\mu}$.

Proposition 2 Given previous assumptions, following rules can be found.

(i) The change direction of the operational risk sensitivity. Given $\ln \frac{\mu}{1-\alpha} \geq 1$, then $\frac{\partial E_{\xi_{w}}}{\partial \xi_{w}} \geq 0$. Given $0<\ln \frac{\mu}{1-\alpha}<1$, then $\frac{\partial E_{\xi_{w}}}{\partial \xi_{w}}<0 ; \frac{\partial E_{\xi_{w}}}{\partial \mu}<0$; $\frac{\partial E_{\mu}}{\partial \xi_{w}}<0, \frac{\partial E_{\mu}}{\partial \mu}<0$;

(ii) The change degree of the operational risk sensitivity:

(1) Given $\left|-\frac{\mu}{\xi_{w}} \ln \frac{\mu}{1-\alpha} \ln \left(\ln \frac{\mu}{1-\alpha}\right)\right| \geq 1$, then $\left|\frac{\partial E_{\xi_{w}}}{\partial \xi_{w}}\right| \geq\left|\frac{\partial E_{\xi_{w}}}{\partial \mu}\right|$, otherwise, $\left|\frac{\partial E_{\xi_{w}}}{\partial \xi_{w}}\right|<\left|\frac{\partial E_{\xi_{w}}}{\partial \mu}\right|$;

(2) Given $\left|\frac{\mu}{\xi_{w}} \ln \frac{\mu}{1-\alpha}\right| \geq 1$, then $\left|\frac{\partial E_{\mu}}{\partial \xi_{w}}\right| \geq\left|\frac{\partial E_{\mu}}{\partial \mu}\right|$, otherwise, $\left|\frac{\partial E_{\mu}}{\partial \xi_{w}}\right|<\left|\frac{\partial E_{\mu}}{\partial \mu}\right|$;

(3) Given $\left|-\ln \frac{\mu}{1-\alpha} \ln \left(\ln \frac{\mu}{1-\alpha}\right)\right| \geq 1$, then $\left|\frac{\partial E_{\xi_{w}}}{\partial \xi_{w}}\right| \geq\left|\frac{\partial E_{\mu}}{\partial \xi_{w}}\right|$,otherwise, $\left|\frac{\partial E_{\xi_{w}}}{\partial \xi_{w}}\right|<\left|\frac{\partial E_{\mu}}{\partial \xi_{w}}\right|$;

(4) Given $\ln \frac{\mu}{1-\alpha} \geq 1$, then $\left|\frac{\partial E_{\xi_{w}}}{\partial \mu}\right| \geq\left|\frac{\partial E_{\mu}}{\partial \mu}\right|$, otherwise, $\left|\frac{\partial E_{\xi_{w}}}{\partial \mu}\right|<\left|\frac{\partial E_{\mu}}{\partial \mu}\right|$.(see proof in Appendix B)

From proposition2(i), as frequency parameter increases, $E_{\xi_{w}}$ and $E_{\mu}$ decreases; As shape parameter increases, $E_{\mu}$ decreases, but $E_{\xi_{w}}$ is uncertain which depends on different operational risk state. Specifically, given $\ln \frac{\mu}{1-\alpha} \geq 1, E_{\xi_{w}}$ increases as frequency parameter 
increases; given $0<\ln \frac{\mu}{1-\alpha}<1, E_{\xi_{w}}$ decreases.

The different influence degree of shape parameter and frequency parameter to $E_{\xi_{w}}$ and $E_{\mu}$ can be determined by four determinants, and therefore we can identify the most important characteristic parameter.

According to proposition 2(ii), let $\frac{\mu}{\xi_{w}} \ln \frac{\mu}{1-\alpha} \ln \left(\ln \frac{\mu}{1-\alpha}\right)=1 \quad, \quad \frac{\mu}{\xi_{w}} \ln \frac{\mu}{1-\alpha}=1$.When $\alpha$ is set at $99.9 \%$, we can get figure2. The point on the curve L1 denotes $\xi_{w}$ and $\mu$ have the same effect on $E_{\xi_{w}}$. Left region of $\mathrm{L}_{1}$ denotes $\xi_{w}$ have less impact than $\mu$, right region of $\mathrm{L}_{1}$ denotes conversely. The point on the curve $\mathrm{L}_{2}$ denotes $\xi_{w}$ and $\mu$ have the same effect on $E_{\mu}$. Left region of $\mathrm{L}_{2}$ denotes $\xi_{w}$ have less impact than $\mu$, while right region of $\mathrm{L}_{1}$ denotes conversely. The curve $L_{1}$ intersects the curve $L_{2}$ at $(0.0151,0.041)$. This point of intersection denotes $\xi_{w}$ and $\mu$ have the same effect on $E_{\xi_{w}}$ and $E_{\mu}$. Obviously, $\mathrm{L}_{1}$ and $\mathrm{L}_{2}$ divide

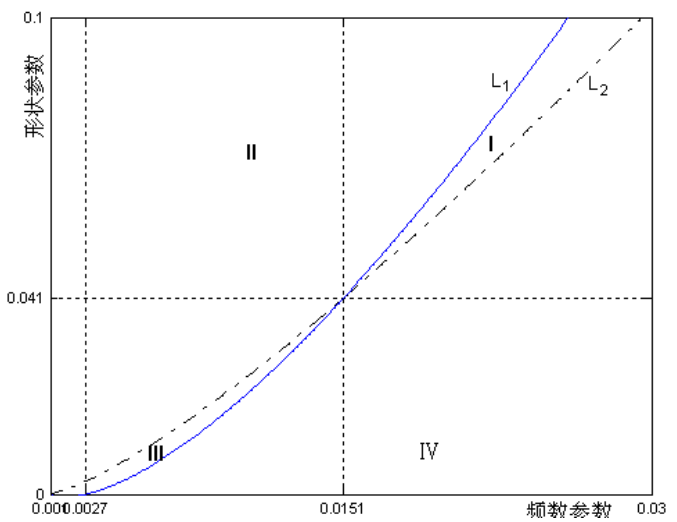

Fig.2. different degree of influence 1

Given $\frac{\mu}{1-\alpha}>1$, then $\mu>0.001$.The figure 2 and figure 3 both regard $\mu=0.001$ as the starting point of abscissa. Key influential indicator for operational risk is different due to different position of $\xi_{w}$ and $\mu$. coordinate plane into four sections I、 П、Ш and $\mathrm{IV}$, different region denoting different state of the operational risk.

Likewise, according to proposition 2(ii) , let $\Delta E_{1}=\ln \frac{\mu}{1-\alpha} \ln \left(\ln \frac{\mu}{1-\alpha}\right), \Delta E_{2}=\ln \frac{\mu}{1-\alpha}$ When setting $\alpha=99.9 \%$, we can get figure3.Curve $\mathrm{L}_{1}^{\prime}$ denotes the different degree which $\xi_{w}$ exerts on $E_{\xi_{w}}$ and $E_{\mu} \cdot \mu=0.0058, \Delta E_{1}=1$ imply the influence degree is the same. Therefore, $\mathrm{L}_{1}^{\prime}$ divide coordinate plane into two different regions: $\Delta E_{1}>1$ and $\Delta E_{1}<1 \quad$.Curve $\mathrm{L}_{2}^{\prime}$ denotes the different degree which $\mu$ exerts on $E_{\xi_{w}}$ and $E_{\mu} \cdot \mu=0.0027 \Delta E_{2}=1$ imply the influence degree is the same. Therefore $\mathrm{L}_{2}^{\prime}$ divides coordinate plane into two different regions: $\Delta E_{2}>1$ and $\Delta E_{2}<1$.

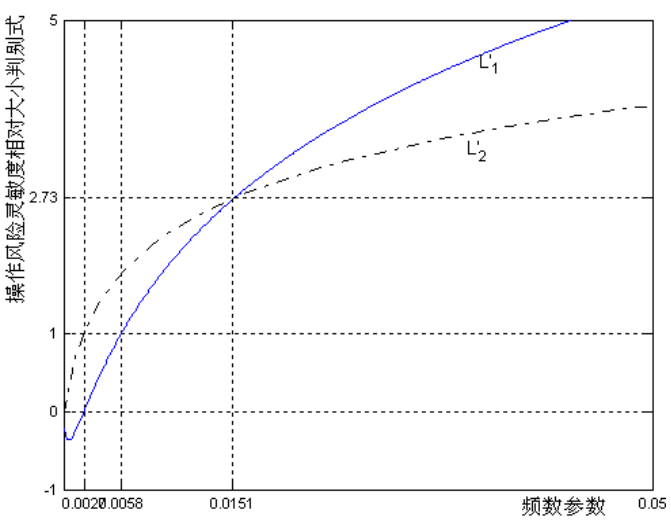

Fig.3. different degree of influence 2

In region I, $\quad\left|\frac{\partial E_{\xi_{w}}}{\partial \xi_{w}}\right|>\left|\frac{\partial E_{\xi_{w}}}{\partial \mu}\right|$ and $\left|\frac{\partial E_{\mu}}{\partial \xi_{w}}\right|<\left|\frac{\partial E_{\mu}}{\partial \mu}\right|$ manifest $\xi_{w}$ is the key influential parameter for $E_{\xi_{w}}$ and $\mu$ is the key influential parameter for $E_{\mu}$.Since in region I, $\mu>0.0151$.Also note 
figure3: $\left|\frac{\partial E_{\xi_{w}}}{\partial \xi_{w}}\right|>\left|\frac{\partial E_{\mu}}{\partial \xi_{w}}\right|$ and $\left|\frac{\partial E_{\xi_{w}}}{\partial \mu}\right|>\left|\frac{\partial E_{\mu}}{\partial \mu}\right|$.

Consequently, we have

$\left|\frac{\partial E_{\xi_{w}}}{\partial \xi_{w}}\right|>\left|\frac{\partial E_{\xi_{w}}}{\partial \mu}\right|>\left|\frac{\partial E_{\mu}}{\partial \mu}\right|>\left|\frac{\partial E_{\mu}}{\partial \xi_{w}}\right| \cdot \xi_{w}$ have the

biggest influence on $E_{\xi_{w}}$.

In region $\Pi,\left|\frac{\partial E_{\xi_{w}}}{\partial \xi_{w}}\right|<\left|\frac{\partial E_{\xi_{w}}}{\partial \mu}\right|$ and $\left|\frac{\partial E_{\mu}}{\partial \xi_{w}}\right|<\left|\frac{\partial E_{\mu}}{\partial \mu}\right|$ manifest $\mu$ is the key influential indicator for $E_{\xi_{w}}$ and $E_{\mu}$, but the influential degree is

diverse. Also note $\mathrm{L}_{2}^{\prime}$ in figure 3:

Suppose $0.001<\mu<0.0027$, we have $\left|\frac{\partial E_{\xi_{w}}}{\partial \mu}\right|<\left|\frac{\partial E_{\mu}}{\partial \mu}\right|$.

That is $\mu$ has little effect on

$E_{\xi_{w}}$ than $E_{\mu}$.As $\mu$ decreases, the diversity increases. Given $\mu>0.0027$, if $\mu$ has the greater effect on $E_{\xi_{w}}$ than $E_{\mu}$.As $\mu$ increases, the diversity increases.

In region III , $\left|\frac{\partial E_{\xi_{w}}}{\partial \xi_{w}}\right|<\left|\frac{\partial E_{\xi_{w}}}{\partial \mu}\right|$ and $\left|\frac{\partial E_{\mu}}{\partial \xi_{w}}\right|>\left|\frac{\partial E_{\mu}}{\partial \mu}\right|$ manifest $\mu$ is the key influential indicator for $E_{\mu}$ and $\xi_{w}$ is the key influential indicator for

$E_{\xi_{w}}$. Since in region III we have $\mu<0.0151$.Also note figure3:

Given $0.001<\mu<0.0027$, then

$$
\left|\frac{\partial E_{\xi_{w}}}{\partial \xi_{w}}\right|<\left|\frac{\partial E_{\mu}}{\partial \xi_{w}}\right| \text { and }\left|\frac{\partial E_{\xi_{w}}}{\partial \mu}\right|<\left|\frac{\partial E_{\mu}}{\partial \mu}\right| \text {. }
$$

Consequently we deduce

$$
\left|\frac{\partial E_{\mu}}{\partial \xi_{w}}\right|>\left|\frac{\partial E_{\mu}}{\partial \mu}\right|>\left|\frac{\partial E_{\xi_{w}}}{\partial \mu}\right|>\left|\frac{\partial E_{\xi_{w}}}{\partial \xi_{w}}\right| \text {,i.e., } \xi_{w} \text { has the }
$$

biggest influence on $E_{\mu} \quad$;Given

$0.0027<\mu<0.0058$, then $\quad\left|\frac{\partial E_{\xi_{w}}}{\partial \xi_{w}}\right|<\left|\frac{\partial E_{\mu}}{\partial \xi_{w}}\right|$ and $\left|\frac{\partial E_{\xi_{w}}}{\partial \mu}\right|>\left|\frac{\partial E_{\mu}}{\partial \mu}\right|\left|\frac{\partial E_{\xi_{w}}}{\partial \mu}\right|<\left|\frac{\partial E_{\mu}}{\partial \mu}\right|$.Consequently we deduce

$$
\left|\frac{\partial E_{\xi_{w}}}{\partial \mu}\right|\left(o r\left|\frac{\partial E_{\mu}}{\partial \xi_{w}}\right|\right)>\left|\frac{\partial E_{\mu}}{\partial \mu}\right|
$$

and $\left|\frac{\partial E_{\xi_{w}}}{\partial \mu}\right|\left(\operatorname{or}\left|\frac{\partial E_{\mu}}{\partial \xi_{w}}\right|\right)>\left|\frac{\partial E_{\xi_{w}}}{\partial \xi_{w}}\right|$. All conclusions manifest that the effect one parameter exerts on the elasticity of another parameter is always greater than the effect that one parameter exerts on its own. Given $0.0058<\mu<0.0151$, then $\left|\frac{\partial E_{\xi_{w}}}{\partial \xi_{w}}\right|>\left|\frac{\partial E_{\mu}}{\partial \xi_{w}}\right|$ and $\left|\frac{\partial E_{\xi_{w}}}{\partial \mu}\right|>\left|\frac{\partial E_{\mu}}{\partial \mu}\right|$.Consequently, we deduce $\left|\frac{\partial E_{\xi_{w}}}{\partial \mu}\right|>\left|\frac{\partial E_{\xi_{w}}}{\partial \xi_{w}}\right|>\left|\frac{\partial E_{\mu}}{\partial \xi_{w}}\right|>\left|\frac{\partial E_{\mu}}{\partial \mu}\right|$,i.e., $\mu$ has the greatest effect on $E_{\xi_{w}}$.

\section{In} region $\mathrm{IV},\left|\frac{\partial E_{\xi_{w}}}{\partial \xi_{w}}\right|>\left|\frac{\partial E_{\xi_{w}}}{\partial \mu}\right|$ and $\left|\frac{\partial E_{\mu}}{\partial \xi_{w}}\right|>\left|\frac{\partial E_{\mu}}{\partial \mu}\right|$ manifest $\xi_{w}$ is the key influential indicator for $E_{\xi_{w}}$ and $E_{\mu}$, but the influence degree is diverse. Note ${ }^{\text {curve }} \mathrm{L}_{1}^{\prime}$ in figure 3: given $0.001<\mu<0.0058$, then $\left|\frac{\partial E_{\xi_{w}}}{\partial \xi_{w}}\right|<\left|\frac{\partial E_{\mu}}{\partial \xi_{w}}\right| . \quad \xi_{w}$ has little effect on $E_{\xi_{w}}$ than $E_{\mu}$. As $\mu$ decreases, the diversity becomes larger. Given $\left|\frac{\partial E_{\xi_{w}}}{\partial \xi_{w}}\right|>\left|\frac{\partial E_{\mu}}{\partial \xi_{w}}\right| \cdot \xi_{w}$ has greater effect on $E_{\xi_{w}}$ than $E_{\mu}$. As $\mu$ increases, the diversity becomes larger.

From the above analysis, if we know the position of the characteristic parameter, we can distinguish key influential factors for $E_{\xi_{w}}$ and $E_{\mu}$ according to proposition 2 . Normally we can predict and supervise the possible variation of the key influential parameters.

Discrimination model of parameter management for operational risk is constructed by proposition 1 and proposition2. For one thing, the model can identify the key influential factor .For another thing, it can predict and 
supervise the possible variation of the key influential parameters.

\subsection{Numerical Analysis}

From the logic process for establishing theoretical model, we know that such model are satisfied under certain condition. We need to check whether the state of the operational risk matches condition before we apply it: First, operational loss severity distribution must be heavy-tailed distribution. When using GEV to comply with loss severity distribution, it is eligible to choose Weibull distribution. If the loss distribution is not heavy-tailed, we can not use the model. Second, after calculating characteristic parameters for loss severity distribution and loss frequency distribution, we should judge whether $\mu /(1-\alpha)>1$ is tenable or not. If the above inequality is satisfies, then we can apply the model, otherwise we cannot use it. Up to now, we do not have professional open database for operational risk to research, due to the confidentiality of the operational loss. Therefore, our data using in empirical analysis is obtained by previous literatures which provide the characteristic parameters for us to test the model. Literature 10 classifies operational loss according to the standard set by the New Basel: Damage to Physical Assets (DPA),Internal Fraud(IF), Employment Practices and Workplace Safety(EPWS),Clients, Products and Business Practices (CPBP), and Execution Fraud (EF), Delivery and Process Management (EDPM), and External Fraud (EF).The literature uses Weibull distribution to comply with operational loss severity distribution and Poisson distribution to comply with operational loss frequency distribution. The obtained characteristic parameters for operational loss distribution are presented in chart 1 as follows.

Weibull distribution is heavy-tailed distribution. When setting $\alpha=99.9 \%$, $\mu /(1-\alpha)>1$ satisfies (as chart1 denotes). Therefore, this case satisfies the condition that model requires and we can use this model to identify the key management parameters for bank. In the following, we set $\alpha=99.9 \%$ and use the data obtained from Wellbull distribution in literature 10 to verify the theory model.

First, test and verify proposition 1 . According to empirical fitting results in literature 10, from proposition1 we can get table1.

Table 1. The sensitivity of $O p \operatorname{VaR}(\alpha)$

\begin{tabular}{ccccc}
\hline $\begin{array}{c}\text { Business } \\
\text { line }\end{array}$ & $\mu$ & $\xi_{w}$ & $\frac{\mu}{1-\alpha}$ & $\Delta E$ \\
\hline IF & 0.52 & 0.59 & 515.9 & -11.44 \\
DPA & 0.3376 & 0.52 & 337.6 & -10.26 \\
EPWS & 0.62 & 0.57 & 617.0 & -11.95 \\
CPBP & 3.24 & $1.3 \mathrm{E}-6$ & 3242.0 & -16.89 \\
EDPM & 9.85 & $3.47 \mathrm{E}-7$ & 9853.5 & -20.40 \\
EF & 141.26 & 0.82 & 141261.1 & -29.33 \\
\hline
\end{tabular}

According to table1, from proposition 1 we know:

(1) The variation direction of the operational VaR. Since $\Delta E<0$, then $E_{\xi_{w}}<0$.Specifically, as shape parameter increases, operational risk value decreases.

(2) The key influential parameters for operational VaR. Discriminant is much greater than1.Consequently, shape parameter exerts much bigger influence on operational risk than frequency parameter. Criterion discriminant $|\Delta E|$ denotes different influence degree of two parameters for operational risk. In six kinds of operational loss, discriminant of $\mathrm{EF}$ is the biggest one, denoting the diversity of influence degree between two parameters is the greatest. However, the discriminant of DPA is the minimum, denoting the diversity of influence degree between two parameters is smallest. That conclusion can be obtained from figure 1 straightforwardly: Given $|\Delta E|$ is greatly than 1, 
it is the loss type EF that locates in the bottom right of the figure, manifesting the diversity of the two characteristic parameter influential degree is the greatest for such loss distribution. It is the loss type DPA that locates in the top left of the figure, manifesting the diversity of the two characteristic parameter influential degree is the smallest.

Next, test and verify proposition2. From table 2 we know,

Table 2. The discriminant of sensitivity formula

\begin{tabular}{ccccc}
\hline Business line & $\left|\frac{\mu}{\xi_{w}} \ln \frac{\mu}{1-\alpha} \ln \left(\ln \frac{\mu}{1-\alpha}\right)\right|$ & $\left|-\ln \frac{\mu}{1-\alpha} \ln \left(\ln \frac{\mu}{1-\alpha}\right)\right|$ & $\ln \frac{\mu}{1-\alpha}$ \\
\hline IF & 10.0 & 5.46 & 11.44 & 6.25 \\
DPA & 6.70 & 3.78 & 10.26 & 5.82 \\
EPWS & 12.9 & 6.95 & 11.95 & 6.42 \\
CPBP & $4.21 \mathrm{E}+07$ & $2.02 \mathrm{E}+07$ & 16.89 & 8.08 \\
EDPM & $5.79 \mathrm{E}+08$ & $2.61 \mathrm{E}+08$ & 20.40 & 9.20 \\
EF & $5.05 \mathrm{E}+03$ & $2.04 \mathrm{E}+03$ & 29.33 & 11.86 \\
\hline
\end{tabular}

(1) The variation direction of operational risk sensitivity. Since $\ln [\mu /(1-\alpha)]>1$, then $\partial E_{\xi_{w}} / \partial \xi_{w}>0$. Specifically, as shape parameter increases, operational risk sensitivity increases

(2) The comparison of changing degree of operational risk sensitivity. All criterion determinants are greater than 1, we can get $\left|\frac{\partial E_{\xi_{w}}}{\partial \xi_{w}}\right|>\left|\frac{\partial E_{\xi_{w}}}{\partial \mu}\right|$ and $\left|\frac{\partial E_{\mu}}{\partial \xi_{w}}\right|>\left|\frac{\partial E_{\mu}}{\partial \mu}\right|$. It is obvious that shape parameter is the key influential parameter for $E_{\xi_{w}}$ and $E_{\mu}$. But the influence degree is different to a certain degree. Specifically, since $\Delta E_{1}>1\left|\frac{\partial E_{\xi_{w}}}{\partial \xi_{w}}\right|>\left|\frac{\partial E_{\mu}}{\partial \xi_{w}}\right|$, we can deduce $\left|\frac{\partial E_{\xi_{w}}}{\partial \xi_{w}}\right|>\left|\frac{\partial E_{\mu}}{\partial \xi_{w}}\right|$.That is to say, shape parameter exerts greater influence on $E_{\xi_{w}}$ than $E_{\mu}$. What's more, as frequency parameter increases, $\Delta E_{1}$ increases and diversity increases. That conclusion can also be obtained from figure 2 and figure 3: In figure2, the coordinate of characteristic parameter for loss distribution locates region IV. In figure 3 , the coordinate of characteristic parameter for loss distribution locates where $\mu>0.0058$. Consequently, shape parameter is the key influence parameter for operational risk sensitivity. Although $\partial E_{\mu} / \partial \xi_{w}<0$, we still have $\partial E_{\xi_{w}} / \partial \xi_{w}>0$. Consequently, as shape parameter increases, operational risk sensitivity increases.

From the above illustrations, we know shape parameter is the key influential parameter for operational risk as well as for operational risk sensitivity: as shape parameter increases, $\operatorname{Op} \operatorname{VaR}(\alpha)$ at risk decreases. And decreasing velocity increases. This reflects that operational risk is increasingly sensitive relating to the change of the shape parameters and shape parameter becomes the key operational risk management parameter. Therefore, in this bank, in order to reduce operational risk, proper measures should be organized and revised to amplify shape parameter. Specifically, management measures like speed up rapid reaction ability, business continuity are expected. In different business line, the influence degree of shape parameter is different. Therefore, the intensity of management measures should be diverse owing to this diversity. To be specific, for business lines which have strong impact on operational risk, management measures should be reinforced extensively. Otherwise, the intensity of management measures should be 
little.

The above examples illustrate that the discrimination model of key management parameters for operational risk is validated and effective. The establish of the discrimination model offers a methodology to identify crucial management parameters. It combines measurement model and management model and make it possible for the integration of the two models. Then operational risk management frameworks to be a complete system. First, from measurement model, we identify key management parameters. Next, input the parameters into management model as management object to come up with measures to manage risks. Then, judge the validity of the management measures by discrimination model as the measures have been enforced for some time: If characteristic parameters of loss distribution changes, the management measures are effective, otherwise, the management measure are invalid. If management measure are effective, we go ahead and analyze whether we achieve our goal, where the error comes from and slightly adjust our management measures. If management measures are invalid, we should find out the reason and amend the management measures. Finally, take revised measures into the management model again. It is likely to integrate measurement model with management model into an operational risk management framework, forming a dynamic system. Therefore, we are able to manage and monitor operational risk dynamically and consequently effectively curb operational risk.

\section{Concluding Remarks}

The existing empirical analysis manifests that operational risk is heavy-tailed. When using Generalized Extreme Value Distribution to fit loss severity distribution, Weibull distribution is a good choice to comply with some kind of operational loss. Based on this, after deducing analytical solution of heavy-tailed operational risk, our article theoretically analyzes sensitivity of $O p \operatorname{VaR}(\alpha)$ and the discrimination model which combines measurement model and management model. Moreover we use examples to illustrate the validity of the model. The establish of the model offers methodology for supervising the effect on the management measures. Further, our theory model combines measurement model and management model, making operational risk management model into a complete framework and forming an dynamic management system of the operational risk. Our research further improves the application of the Loss Distribution Approach in operational risk measurement and management.

\section{Reference}

R. Kennet. How to Introduce an Effective Operational Risk Management Framework. In Robert Hübner (ed.) Operational Risk: Firm-wide Issues for Financial Institutions[M]. London: a division of Risk Waters Group Ltd., 2003:73-92.

A. Peccia. Using operational risk models to manage operational risk. In C. Alexander (ed.), Operational Risk: Regulation, Analysis and Management[M]. London: Pearson Education Limited,2003:262-284.

Basel Committee on Banking Supervision. International Convergence of Capital Measurement and Capital Standards: A Revised Framework[S], Bank for International Settlements, 2004.6.

C. Alexander. Bayesian Methods for Measuring Operational Risk[R]. ICMA Centre Discussion Papers in Finance icma, Henley Business School, Reading University. 2000.

C. Alexander. Managing operational risks with Bayesian networks. In C. Alexander (ed.), Operational Risk: Regulation, Analysis and Management[M]. London: Pearson Education Limited, 2003:285-295.

R. G. Cowell, R. J.Verrall and Y. K. Yoon. Modelling Operational Risk With Beyesian Networks[J]. Journal of Risk and Insurance, 2007,74(4):795-827.

M. Neil, N. E. Fenton and M. Tailor. Using Bayesian Networks to Model Expected and Unexpected Operational Losses[J]. Risk Analysis, 2005, 25(4): 1539-6924.

L. Dalla Valle and P. Giudicib. A Bayesian Approach to Estimate the Marginal Loss Distributions in Operational Risk Management[J]. Computational Statistics \& Data Analysis, 2008,52(6): 3107-3127.

SHI Dao-ji. Applied method of extremes statistics[M]. Tianjin: Tianjin Science and Technology Press, 
2006.

Dionne and H. Dahen. What about Underevaluating Operational Value at Risk in the Banking Sector?(C), The 6th Annual Premier Global Event on ERM, 2008.

A. Chapelle, Y. Crama, G. Hübner and J.-P. Peters. Practical Methods for Measuring and Managing Operational Risk in the Financial Sector: A Clinical Study [J], Journal of Banking \& Finance. 2008,32(6):1049-1061.

J.D. Opdyke, DataMineit, LLC. Estimating Operational Risk Capital with Greater Accuracy, Precision, and Robustness[J].Journal of Operational Risk , June 2014 ,9(4)

K. Bocker and C. K1Äuppelberg. Operational VaR: a Closed-Form Approximation [J], Risk of London,2005,18(12):90-93.

K. Bocker and J. Sprittulla. Operational VAR: meaningful means[J]. Risk of London,2006,19(12):96-98.

K. Bocker. Operational risk analytical results when high-severity losses follow a generalized Pareto distribution (GPD)[J]. Risk of London, 2006,8(4):117-120.

Ames Mark, Schuermann Til, Scott. Hal S. Bank capital for operational risk: A tale of fragility and instability[J]. Journal of Risk Management in Financial Institutions 2015,8(3) pp. 227-243(17)

MO Jian-ming and Zhou Zong-fang. Confidence Interval and Sensitivity of the operational VaR in LDA[J], Systems engineering, 2007,25(10): 33-39.

S é verine Plunus, Georges Hü bner, Jean-Philippe Peters.Measuring operational risk in financial institutions[J]. Applied Financial Economics ,September2012.22(18):1553-1569

\section{Appendix:}

\section{A: proof of proposition1 :}

proof

know $\left._{\left(\ln \frac{\mu}{1-\alpha}\right.}\right)^{\frac{1}{\xi_{w}}} \geq 0$ and $\xi_{w}>0$. When $0<\ln \frac{\mu}{1-\alpha} \leq 1, E_{\xi_{w}} \geq 0$, When $\ln \frac{\mu}{1-\alpha}>1, E_{\xi_{w}}<0$.Since $\ln \frac{\mu}{1-\alpha} \geq 0$ an $\mathrm{d} \xi_{w}>0$, then $_{E_{\mu}}=\left(\xi_{w} \ln \frac{\mu}{1-\alpha}\right)^{-1}>0$.

For(ii),from equation(5) we know, $\lim _{\alpha \rightarrow 1} E_{\xi_{w}}=\lim _{\alpha \rightarrow 1}\left[-\ln \left(\ln \frac{\mu}{1-\alpha}\right)^{\xi_{w}^{-1}}\right]=-\infty$.From equation(6) we know, $\lim _{\alpha \rightarrow 1} E_{\mu}=\lim _{\alpha \rightarrow 1}\left(\xi_{w} \ln \frac{\mu}{1-\alpha}\right)^{-1}=0$.

For(iii), since $\frac{E_{\xi_{w}}}{E_{\mu}}=\frac{-\ln \left(\ln \frac{\mu}{1-\alpha}\right)^{\xi_{w}^{-1}}}{\left(\xi_{w} \ln \frac{\mu}{1-\alpha}\right)^{-1}}=-\ln \frac{\mu}{1-\alpha} \ln \left(\ln \frac{\mu}{1-\alpha}\right)$. When $\quad\left|\ln \frac{\mu}{1-\alpha} \ln \left(\ln \frac{\mu}{1-\alpha}\right)\right| \geq 1, \quad\left|E_{\xi_{w}}\right| \geq\left|E_{\mu}\right|$, otherwise, $\left|E_{\xi_{w}}\right|<\left|E_{\mu}\right|$.

\section{B: proof of proposition2:}

proof Firstly we prove proposition(i).From equation(5) we know that:

$$
\begin{aligned}
& \frac{\partial E_{\xi_{w}}}{\partial \xi_{w}}=\xi_{w}^{-2} \ln \left(\ln \frac{\mu}{1-\alpha}\right) \\
& \frac{\partial E_{\xi_{w}}}{\partial \mu}=-\left(\xi_{w} \mu \ln \frac{\mu}{1-\alpha}\right)^{-1}
\end{aligned}
$$

Since $\xi_{w}>0, \ln \frac{\mu}{1-\alpha} \geq 0$, and $\mu>0$,from equation (7) we know,when $\ln \frac{\mu}{1-\alpha} \geq 1, \frac{\partial E_{\xi_{w}}}{\partial \xi_{w}} \geq 0$. When $0<\ln \frac{\mu}{1-\alpha}<1, \frac{\partial E_{\xi_{w}}}{\partial \xi_{w}}<0$.From equation(8)we know $\frac{\partial E_{\xi_{w}}}{\partial \mu}<0$.

From equation(6) we have: 


$$
\begin{gathered}
\frac{\partial E_{\mu}}{\partial \xi_{w}}=-\xi_{w}^{-2}\left(\ln \frac{\mu}{1-\alpha}\right)^{-1} \\
\frac{\partial E_{\mu}}{\partial \mu}=-\xi_{w}^{-1} \mu^{-1}\left(\ln \frac{\mu}{1-\alpha}\right)^{-2}
\end{gathered}
$$

Since $\xi_{w}>0, \ln \frac{\mu}{1-\alpha} \geq 0, \mu>0$, From equation (10) we know $\frac{\partial E_{\mu}}{\partial \xi_{w}}<0$.

For proposition (ii), from $\frac{\partial E_{\xi_{w}}}{\partial \xi_{w}} / \frac{\partial E_{\xi_{w}}}{\partial \mu}=-\frac{\mu}{\xi_{w}} \ln \frac{\mu}{1-\alpha} \ln \left(\ln \frac{\mu}{1-\alpha}\right)$ we know that given $\left|-\frac{\mu}{\xi_{w}} \ln \frac{\mu}{1-\alpha} \ln \left(\ln \frac{\mu}{1-\alpha}\right)\right| \geq 1$, we have $\left|\frac{\partial E_{\xi_{w}}}{\partial \xi_{w}}\right| \geq\left|\frac{\partial E_{\xi_{w}}}{\partial \mu}\right|$, otherwise, $\left|\frac{\partial E_{\xi_{w}}}{\partial \xi_{w}}\right|<\left|\frac{\partial E_{\xi_{w}}}{\partial \mu}\right|$.

From $\frac{\partial E_{\mu}}{\partial \xi_{w}} / \frac{\partial E_{\mu}}{\partial \mu}=\frac{\mu}{\xi_{w}} \ln \frac{\mu}{1-\alpha}$ we know that, since $\left|\frac{\mu}{\xi_{w}} \ln \frac{\mu}{1-\alpha}\right| \geq 1,\left|\frac{\partial E_{\mu}}{\partial \xi_{w}}\right| \geq\left|\frac{\partial E_{\mu}}{\partial \mu}\right|$, otherwise, $\left|\frac{\partial E_{\mu}}{\partial \xi_{w}}\right|<\left|\frac{\partial E_{\mu}}{\partial \mu}\right|$.

$$
\text { From } \frac{\partial E_{\xi_{w}}}{\partial \xi_{w}} / \frac{\partial E_{\mu}}{\partial \xi_{w}}=-\ln \frac{\mu}{1-\alpha} \ln \left(\ln \frac{\mu}{1-\alpha}\right) \text { we know that given }\left|-\ln \frac{\mu}{1-\alpha} \ln \left(\ln \frac{\mu}{1-\alpha}\right)\right| \geq 1,\left|\frac{\partial E_{\xi_{w}}}{\partial \xi_{w}}\right| \geq\left|\frac{\partial E_{\mu}}{\partial \xi_{w}}\right|,
$$
otherwise, $\left|\frac{\partial E_{\xi_{w}}}{\partial \xi_{w}}\right|<\left|\frac{\partial E_{\mu}}{\partial \xi_{w}}\right|$.

From $\frac{\partial E_{\xi_{w}}}{\partial \mu} / \frac{\partial E_{\mu}}{\partial \mu}=\ln \frac{\mu}{1-\alpha}$ we know that given $\ln \frac{\mu}{1-\alpha} \geq 1,\left|\frac{\partial E_{\xi_{w}}}{\partial \mu}\right| \geq\left|\frac{\partial E_{\mu}}{\partial \mu}\right|$,otherwise, $\left|\frac{\partial E_{\xi_{w}}}{\partial \mu}\right|<\left|\frac{\partial E_{\mu}}{\partial \mu}\right|$. 